\title{
Anforderungen an die 5G-Kommunikation für die Automatisierung in vertikalen Domänen
}

\author{
Michael Bahr, Joachim Walewski \\ Siemens AG \\ Corporate Technology \\ Research in Digitalization and Automation \\ Internet of Things \\ Otto-Hahn-Ring 6 \\ 81739 München \\ bahresiemens.com \\ joachim.walewski@siemens.com
}

\begin{abstract}
Zusammenfassung. Dieser Beitrag berichtet über den aktuellen Stand der Standardisierung von Anforderungen für die fünfte Generation von Mobilfunknetzen (5G) in der 3GPP Arbeitsgruppe SA1 als auch anstehende Aktivitäten in der nahen Zukunft. Hierbei liegt der Schwerpunkt auf industriellen Anforderungen. Weiterhin wird kurz auf die Vergabe von Frequenzbändern für lokale 5G-Netze eingegangen.
\end{abstract}

\section{$1 \quad$ Einleitung}

Die fünfte Generation der Mobilfunknetze (5G) behandelt drei große Anwendungsgebiete: weiterentwickeltes mobiles Breitband für $\mathrm{Gb} / \mathrm{s}$-Datenraten für beispielsweise Videos (enhanced mobile broadband, eMBB), massive Maschinenkommunikation für die Anbindung sehr vieler Geräte im Internet der Dinge (massive machine-type communication, mMTC), und hochzuverlässige Maschinenkommunikation für anspruchsvolle, industrielle Anwendungen (ultra-reliable machine-type communication, uMTC). 5G hat somit als erste Mobilfunkgeneration ausdrücklich den Anspruch, auch Kommunikation für Kontrollschleifen im industriellen Umfeld zur Verfügung zustellen. uMTC wird im 3GPP-Kontext oft auch ultra-reliable low-latency communication bezeichnet (URLLC).

\section{Genereller Überblick zur 5G-Standardisierung}

5G-Mobilfunknetze werden gegenwärtig in der Third Generation Public Partnership (3GPP) in den Freigaben 15 und 16 standardisiert, [3G18]. Diese Freigaben, auch Releases genannt, laufen im allgemeinen zeitversetzt und überlappend parallel ab, und es wird ein stufenweiser Ansatz von Dienstanforderungen über die Architektur zu Technologiespezifikation verfolgt. Freigabe 15, die erste 5G-Spezifikation, wird Ende 2018 
im Wesentlichen abgeschlossen sein, ebenfalls die Sammlung der Dienstanforderungen für Freigabe 16.

\section{$3 \quad 5 G$ Dienstanforderungen für uMTC im industriellen Umfeld}

5G-Dienstanforderungen für uMTC im industriellen Umfeld wurden im 3GPPTechnical-Report TR 22.804 ,Study on Communication for Automation in Vertical Domains“ zusammengetragen [3G18b]. Diese Studie wurde bei der achtzigsten Plenarversammlung der 3GPP im Juni 2018 verabschiedet. TR 22.804 enthält eine umfangreiche Sammlung von Anwendungsfällen, Kenngrößen und Einflussgrößen. Die betrachteten Anwendungsfälle stammen nicht nur aus der Industrieautomatisierung, sondern zum Beispiel auch aus der Energieautomatisierung und dem Personennahverkehr. Auch die Unterhaltungsindustrie steuerte URLLC-Anwendungsfälle bei.

Neben TR 22.804 wurden in weiteren Studien und Spezifikationen Themen und 5GDienstanforderungen adressiert, die ebenfalls für uMTC im industriellen Umfeld von Bedeutung sind. Abbildung 1 gibt einen Überblick über diese Dokumente und wie sie aufeinander aufbauen.

Es ist zu beachten das technische Berichte, also Technical Reports (TR), nur einen informativen aber keinen normativen Charakter haben. Sie sind oft Vorarbeiten für die normativen technischen Spezifikationen (TS).

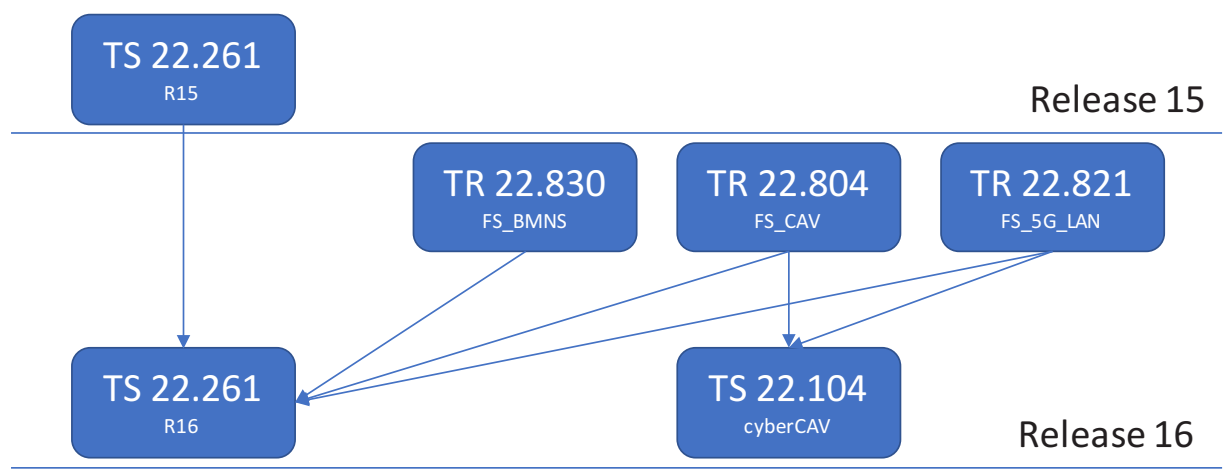

Abb. 1. 3GPP-Dokumente für 5G-Dienstanforderungen für uMTC im industriellen Umfeld (Freigabe 16)

Der im März 2018 verabschiedete 3GPP-Technical-Report TR 22.821 „Feasibility Study on LAN Support in 5G" adressiert unter anderem die Unterstützung von EchtzeitEthernet-Diensten über 5G [3G18c]. Inhalt dieser Studie sind Überlegungen, wie 5GNetze ohne die Verwendung von IP-Tunneln und Kommunikationsprotokollumsetzern direkt an sogenannte TSN-Netze (time synchronized networks) angeschlossen werden können. TSN-Netze sind Ethernet-basierte Kommunikationsnetze mit hochgenauer Zeitsynchronisation. 
Bei der achtzigsten Plenarversammlung der 3GPP wurde auch ein Antrag auf die Erarbeitung von normativen Anforderung für cyber-physikalische Kontrollanwendungen verabschiedet. Das zugehörige Projekt läuft unter der Abkürzung cyberCAV und soll Ende 2018 abgeschlossen sein [3G18e]. Kommunikation und Kommunikationsanwendungen in der produzierenden Industrie werden hierbei besonders hervorgehoben. Die erarbeiteten normativen Anforderungen basieren sowohl auf in TR 22.804 [3G18b] und TR 22.821 [3G18c] identifizierten Anforderungen. Die normativen Anforderungen werden sowohl in eine neue Version des Standards TS 22.261 „Service requirements for the 5G system“ für Freigabe 16 [3G18a] als auch in einen neuen Standard, nämlich TS 22.104 ,Service requirements for cyber-physical control applications in vertical domains" eingehen [3G18f].

Der Standard TS 22.261 „Service requirements for the 5G system“ [3G18a] ist das Hauptdokument für die Dienstanforderungen an 5G-Netze. 5G-Dienstanforderungen, die innerhalb der Arbeiten für uMTC im industriellen Umfeld erarbeitet wurden, aber auch für andere Anwendungsgebiete von $5 \mathrm{G}$ von Bedeutung sind oder einen allgemeinen Charakter haben, fließen in diesen Standard TS 22.261 ein. 5G-Dienstanforderungen, die ihren Schwerpunkt bei uMTC im industriellen Umfeld beziehungsweise in vertikalen Domänen haben, fließen in den speziellen Standard TS 22.104 ein.

Parallel zu den obigen Aktivitäten wurde auch eine Studie zu Geschäftsrollen im Zusammenhang mit „Network Slicing“ von der 3GPP erarbeitet: TR 22.830 „Study on Business Role Models for Network Slicing“ [3G18d]. Zwei für die produzierende Industrie wichtige Szenarieren adressieren hierin das nahtlose Verfolgen und Überwachen von Wertschöpfungsketten zwischen Fabriken und der problemlose Wechsel von einem Kommunikationsanbieter zum anderen. Aus TR 22.830 abgeleitete Dienstanforderungen werden ebenfalls in TS 22.261 eingebracht werden [3G18g].

\section{$4 \quad$ Erkenntnisse aus der Erarbeitung von TR 22.804}

5G erfordert mit seinem erklärten Ziel, Mobilfunk auch in vertikalen Domänen und für ultra-reliable low-latency communication anwendbar zu machen, zum ersten Mal eine aktive Teilnahme aus dem industriellen Umfeld und anderen vertikalen Domänen an der 5G-Standardisierung der 3GPP, insbesondere bei der Definition der Anforderungen und Kennzahlen in der 3GPP Arbeitsgruppe SA1. Der dadurch mögliche Austausch ist notwendig, um eine hohe Anwendbarkeit von 5G-Netzen im industriellen Umfeld und in anderen vertikalen Domänen zu erreichen.

Neben den Erkenntnissen über die Arbeitsweise der 3GPP und der Abläufe in der 3GPP-Standardisierung traten auch etliche inhaltliche Themen als wichtige Erkenntnisse zu industriellen Anforderungen an 5G-Netze hervor. Diese inhaltlichen Erkenntnisse sind in den folgenden Abschnitten kurz erläutert. 


\subsection{Gemeinsamkeiten über „vertikale Grenzen“ hinweg}

Wie zu erwarten zeigte sich beim Zusammentragen von Anwendungsfällen aus verschiedenen Anwendungsfeldern, dass Anforderungen für die Kontrolle von physikalischen Prozessen sehr ähnliche Anforderungen an die Kommunikation zur Folge haben. Während der ursprüngliche Fokus der 3GPP auf kurzen Latenzzeiten (also unter $10 \mathrm{~ms}$ ) lag, zeigte sich im Rahmen von TR 22.804 dass zwei andere Klassen von Anforderungen allen Anwendungen gemeinsam und somit viel wichtiger sind, nämlich die Zuverlässigkeit [IE09] als auch die Sicherheit (siehe auch Abschnitte 4.2 und 4.3 zu Sicherheit).

Zum Thema Zuverlässigkeit wurden hohe Verfügbarkeiten (typischerweise 99,99\% und höher) als auch hohe Funktionssicherheiten identifiziert. Letztere zeigen sich in den Anforderungen an die mittlere Zeit zwischen Kommunikationsdienstfehlern von mindestens einer Woche und im Extremfall von zehn Jahren.

Interessanterweise stellte sich heraus, dass ähnlich Anforderungen auch für die digitale Medienproduktion gelten, zum Beispiel bei der Verwendung von drahtlose Mikrofonen und Kameras.

\subsection{Kennzahlen (insbesondere Latenzzeiten) sind nicht alles}

In 5G-Diskussionen wurde anfänglich davon ausgegangen, dass die Unterstützung von kurzen Latenzzeiten (Stichwort: $1 \mathrm{~ms}$ ) hinreichend für die Unterstützung von Automatisierungsanwendungen ist. Dank TR 22.804 wurde nicht nur klar, dass andere Kenngrößen wie Verfügbarkeit und Zuverlässigkeit mindestens genauso wichtig sind (siehe Abschnitt 4.1), sondern dass Automatisierungsanwendungen noch eine Vielzahl anderer Anforderungen haben.

Bei der Latenz zum Beispiel wurde schnell klar, dass statistische Kennzahlen mit Konfidenzintervallen nicht ausreichen. Stattdessen werden Kennzahlen für die maximal erlaubte Latenz angegeben, im Sinne der in der Industriekommunikation oft verwendeten deadline für die Übertragungszeit. Aus diesem Grund werden auch keine Kennzahlen für das Jitter, einer beliebten statistischen Kennzahl in Telekommunikationsnetzen, angegeben. Stattdessen wird das Prinzip der Rechtzeitigkeit verwendet [3G18f].

Weitere wichtige Anforderungsbereiche sind nicht-öffentlicher Betrieb (siehe Abschnitt 4.2), Sicherheit, Überwachung der Dienstgütequalität (Quality-of-Service Monitoring) und die Integration von Ethernet-basierten und 5G industriellen Netzen.

Für den Anforderungsbereich Sicherheit zeichneten sich mehrere wichtige Themen $\mathrm{ab}$ :

- Nicht-öffentliche 5G-Netze sollen auch andere Zertifikate als AKA' und 5GAKA zulassen. Netzbetreiber werden Geräten, die andere Zertifikate als AKA'und 5G-AKA benutzen, keinen Zugang zu öffentlichen Netzen erlauben. 
- Die Automatisierungsanwendung soll überwachen können, ob und wie Verschlüsselung im 5G-Netz aktiviert ist. Dies ist typischerweise für drei Anwendungsklassen wichtig: Kommunikation mit ultrakurzen Latenzzeiten, batteriebetriebene Geräte, und Automatisierungsfunktionen in Edge-Clouds.

- Verschlüsselung auf der Anwendungsebene darf von 5G-Netzen nicht blockiert werden. Dies ist vor allem bei Kommunikationsdiensten mit kurzen Latenzzeiten von Bedeutung.

Die Überwachung der Dienstgütequalität, das sogenannte Quality-of-Service- oder QoS-Monitoring, erlangt seine große Bedeutung aus zwei wichtigen Anforderungsbereichen:

- Schnelle Erkennung und Lokalisierung von Kommunikationsproblemen im industriellen 5G-Netz. Da reale Industrie- und Bearbeitungsprozesse gesteuert werden, kann eine Verletzung der Dienstgütequalität zu kostspieligen Stillstandszeiten führen, im Ernstfall sogar zu Schäden an den Produktionsmaschinen.

- Überwachung von Service Level Agreements, die zwischen dem Nutzer eines industriellen 5G-Netzes wie einer Fabrik und dem Betreiber des industriellen 5G-Netzes vereinbart wurden.

Die Einbindung von industriellen 5G-Netzen in bestehende und zukünftige Ethernetbasierte industrielle Kommunikationsnetze sind Anforderungen in TR 22.821 und TS 22.104 definiert. Neben der Bereitstellung von „Ethernet-Diensten“ ist hierbei die Zeitsynchronisation ein wichtiges Thema.

\subsection{Industrielle 5G-Netze als nicht-öffentliche Netze}

Es zeichnete sich früh $a b$, dass für ein besseres Verständnis für die Umsetzung der stringenten industriellen Anforderungen auch die Frage der Aufstellung, des Einsatzes und der Organisation von 5G-Netzen betrachtet und mit entsprechenden Anforderungen beschrieben werden muss.

Industrielle 5G-Netze sind ein Beispiel für nicht-öffentliche Mobilfunknetze. Der Begriff nicht-öffentliches Netz wurde im Verlauf der Standardisierungsaktivitäten von TR 22.804 (FS_CAV) und TS 22.104 (cyberCAV) entwickelt, um die besonderen Anforderungen bezüglich Aufstellung, Einsatz und Organisation industrieller 5G-Netze beschreiben und umsetzen zu können. Nicht-öffentliche Netze werden im Prinzip Zugriff auf alle 5G-Funktionalitäten haben, also auch Netzwerk-Slices.

Verschiedene Aspekte, die für den Einsatz industrieller 5G-Netze wichtig sind und zum Teil miteinander verwoben sind, können mit nicht-öffentlichen Netzen umgesetzt werden:

- Abgrenzung der Netze: Um die stringenten industriellen Anforderungen wie sehr geringe Latenz und sehr hohe Zuverlässigkeit zu erfüllen, müssen industrielle von anderen Netzen, wie zum Beispiel öffentlichen 5G-Mobilfunknetzen, gut abgegrenzt sein. 
- Anzahl der Netze: Industrielle 5G-Netze werden oft nur eine lokale Ausdehnung haben, zum Beispiel ein Fabrikgelände oder Teile davon. Es wird erwartet, dass es in Zukunft eine sehr große Anzahl solcher nicht-öffentlichen Netze mit unterschiedlichsten Betreibern geben wird, die alle eine eigene Netzkennung benötigen. Mit den bisherigen Konzepten aus den öffentlichen Netzen ist das nur sehr schwierig möglich.

- Sicherheitszertifikate der Netze: Neben den gängigen 3GPP-Zertifikaten $\mathrm{AKA}^{\prime}$ und 5G-AKA dürfen in diesen Netzen auch andere Zertifikate verwendet werden, beispielsweise TLS- und X.509-Zertifikate. Werden 3GPPZertifikate benutzt, können vereinbarte Kommunikationsdienste von $5 \mathrm{G}-\mathrm{Ge}-$ räten des nicht-öffentlichen Netzes auch im öffentlichen 5G-Netz genutzt werden, wenn ein entsprechender Vertrag mit dem öffentlichen Netz vorliegt. Dies ist beispielsweise für die Verfolgung und Überwachung von Waren im Transit zwischen Produktionsstandorten von Interesse. Werden andere Zertifikate benutzt, ist solch ein Übergang zwischen nicht-öffentlichen und öffentlichen Netzen nicht möglich. Nur ausschließlich vom Fabrikbetreiber autorisierte 5G-Geräte haben Zugang zu solch einem nicht-öffentlichen Netz.

- Betreiber der Netze: Vielfältige Betreibermodelle für eine flexible und bedarfsgerechte Nutzung industrieller 5G-Netze, die die Anforderungen von URLLC erfüllt, sind mit nicht-öffentlichen Netzen möglich. Zum einen können Fabriken ihre industriellen 5G-Kommunikationsnetze selber betreiben, zum anderen kann der Betrieb eines solchen Netzes auch an Kommunikationsfirmen ausgelagert werden, wie zum Beispiel an Mobilfunkbetreiber, die dann das nicht-öffentliche Netz im Auftrag der Fabrik nach deren Vorgaben betreiben.

An der Definition von öffentlichen Netzen musste nichts geändert werden. Als zusätzliche Anforderung wurde jedoch stipuliert, dass öffentliche Netze prinzipiell nichtöffentliche Netze mit 3GPP-Zertifikaten als virtualisierte Teilnetze unterstützen können müssen.

\subsection{Aufstellung industrieller 5G-Netze}

Ein ebenfalls wichtiger Punkt in Zusammenhang mit nicht-öffentlichen Netzen (siehe Abschnitt 4.2) ist die Aufstellung und die Art des Einsatzes von industriellen 5GNetzen. Die folgenden drei Kategorien haben sich herauskristallisiert:

- $\quad$ Eigenständiges 5G-Netz

- Virtualisiertes Teilnetz, zum Beispiel als eine oder mehrere Network-Slices in einem öffentlichen Netz

- Teil eines 5G-Netzes, zum Beispiel eine drahtlose 5G-Verbindung in einem Teilbereichen einer Fabrik 


\subsection{Ende-zu-Ende-Dienste für Kontrollanwendungen sind ein neues Feld}

Sowohl für 3GPP als auch für die industrielle Automatisierung sind Ende-zu-EndeDienste für Kontrollanwendungen ein neues Feld.

Während der Erarbeitung von TR 22.804 stellte sich zum Thema Dienste folgendes heraus: Zum einen hat 3GPP kein normiertes Referenzmodell von Dienstbeschreibungen. Es ist also nicht klar, welche Aspekte eines Dienstes zwingend beschrieben werden müssen. Außerdem stellte sich heraus, dass Kommunikationsdienste oftmals durch Netzwerk- und nicht Dienstanforderungen beschrieben werden. Letzteres hat zur Folge, dass in diesen Fällen keine zwingenden Ende-zu-Ende Anforderungen aus den 3GPPDokumenten ableitbar sind. Zum anderen werden auch in der industriellen Automatisierung oftmals Dienstanforderungen entweder durch reine Anwendungsbeschreibungen (Stichwort: zyklische Anwendungen) oder durch Netzwerkanforderungen oder durch eine stark integrierte Kombination von beiden beschrieben.

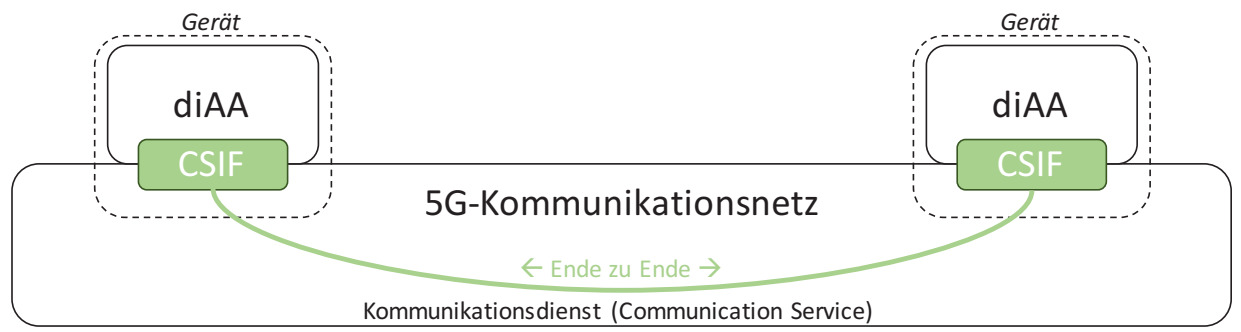

diAA - distributed automation application/function (verteilte Automationsanwendung / - funktion) CSIF - Communication Service Interface (Kommunikationsdienstschnittstelle, Referenzschnittstelle)

Abb. 2. Ende-zu-Ende-Sicht von Kommunikationsdiensten

Zur Lösung dieser Probleme wurden die Anforderungen in TR 22.804 aus einer für Dienste adäquaten Ende-zu-Ende Sicht formuliert. Abbildung 2 zeigt das zugrundeliegende allgemeine Konzept. Ende-zu-Ende bedeutet hier, von der Schnittstelle CSIF zwischen Applikation und 5G-Netz am Anfang des Kommunikationsdienstes bis zur Schnittstelle CSIF zwischen 5G-Netz und Applikation am Ende des Kommunikationsdienstes. Wo nicht vorhanden wurden die entsprechenden Kenngrößen und Einflussgrößen neu in 3GPP eingeführt. Beispiele sind hierfür die Ende-zu-Ende-Latenzzeit und das Transferintervall.

Tabelle 1. Vorgeschlagene Kenngrößen für die Beschreibung der Schnittstelle von zuverlässigen Kommunikationsdiensten [3G18b]

\begin{tabular}{|c|c|c|c|c|}
\hline \multirow[t]{2}{*}{ Parameter } & \multirow{2}{*}{$\begin{array}{c}\text { Typische Größe } \\
\text { (Einheit) }\end{array}$} & \multicolumn{3}{|c|}{ Verkehrsklasse / Art der Kommunikation } \\
\hline & & $\begin{array}{c}\text { Deterministisch } \\
\text { periodisch }\end{array}$ & $\begin{array}{l}\text { Deterministisch } \\
\text { a-periodische }\end{array}$ & $\begin{array}{l}\text { Nicht-deter- } \\
\text { ministisch }\end{array}$ \\
\hline $\begin{array}{l}\text { Verfügbarkeit } \\
\text { des Kommu- } \\
\text { nikations- } \\
\text { dienstes. }\end{array}$ & $\begin{array}{l}\text { Minimale Verfüg- } \\
\text { barkeit } \\
\text { (dimensionslos) }\end{array}$ & $\mathrm{X}$ & $\mathrm{X}$ & $\mathrm{X}$ \\
\hline
\end{tabular}




\begin{tabular}{|c|c|c|c|c|}
\hline \multirow[t]{2}{*}{ Parameter } & \multirow{2}{*}{$\begin{array}{l}\text { Typische Größe } \\
\text { (Einheit) }\end{array}$} & \multicolumn{3}{|c|}{ Verkehrsklasse / Art der Kommunikation } \\
\hline & & $\begin{array}{c}\text { Deterministisch } \\
\text { periodisch }\end{array}$ & $\begin{array}{l}\text { Deterministisch } \\
\text { a-periodische }\end{array}$ & $\begin{array}{c}\text { Nicht-deter- } \\
\text { ministisch }\end{array}$ \\
\hline $\begin{array}{l}\text { Ende-zu-Ende } \\
\text { Latenzzeit }\end{array}$ & Maximalwert (s) & $\mathrm{X}$ & $\mathrm{X}$ & $\mathrm{X}$ \\
\hline $\begin{array}{l}\text { Zeit zwischen } \\
\text { Kommunika- } \\
\text { tionsdienst- } \\
\text { fehlern }\end{array}$ & $\begin{array}{l}\text { Mittlere Zeit zwi- } \\
\text { schen Kommuni- } \\
\text { kationsdienstfeh- } \\
\text { lern (s) }\end{array}$ & $\mathrm{X}$ & $\mathrm{X}$ & $\mathrm{X}$ \\
\hline Dienstbitrate & $\begin{array}{l}\text { Zielrate }(\mathrm{b} / \mathrm{s}) \\
\text { Minimalrate }(\mathrm{b} / \mathrm{s}) \\
\text { Zeitfenster }(\mathrm{s})\end{array}$ & - & $\mathrm{X}$ & $\mathrm{X}$ \\
\hline $\begin{array}{l}\text { Aktualisier- } \\
\text { ungszeit }^{1}\end{array}$ & $\begin{array}{l}\text { Ziel-Aktualisie- } \\
\text { rungszeit (s); } \\
\text { maximale Erneue- } \\
\text { rungszeit (s) }\end{array}$ & $\mathrm{X}$ & - & - \\
\hline
\end{tabular}

Außerdem wurden geeignete Kenngrößen (Tabelle 1) und Einflussgrößen (Tabelle 2) für die Beschreibung von zuverlässigen $5 \mathrm{G}$-Kommunikationsdiensten eingeführt. Es wurde auch identifiziert, für welche der drei typischen Automatisierungsverkehrsklassen diese Parameter Verwendung finden (X - trifft zu; (-) - trifft typischerweise nicht $\mathrm{zu},-$ - trifft nicht $\mathrm{zu})$.

Tabelle 2. Vorgeschlagene Einflussgrößen für die Beschreibung von Schnittstellen zuverlässiger 5G-Kommunikationsdienste [3G18b].

\begin{tabular}{|c|c|c|c|c|}
\hline \multirow[t]{2}{*}{ Parameter } & \multirow{2}{*}{$\begin{array}{c}\text { Typische Größe } \\
\text { (Einheit) }\end{array}$} & \multicolumn{3}{|c|}{ Verkehrsklasse / Art der Kommunikation } \\
\hline & & $\begin{array}{c}\text { Deterministisch } \\
\text { periodisch }\end{array}$ & $\begin{array}{c}\text { Deterministisch } \\
\text { a-periodische }\end{array}$ & $\begin{array}{c}\text { Nicht-deter- } \\
\text { ministisch }\end{array}$ \\
\hline Burst & $\begin{array}{l}\text { Spitzenbitrate } \\
\text { (b/s); Maximale } \\
\text { Burstlänge (s) } \\
\end{array}$ & - & $\mathrm{X}$ & $\mathrm{X}$ \\
\hline Überlebenszeit $^{2}$ & Maximum (s) & $\mathrm{X}$ & $\mathrm{X}$ & - \\
\hline $\begin{array}{l}\text { Größe der Auto- } \\
\text { matisierungs- } \\
\text { botschaft }\end{array}$ & Maximum (B) & $\mathrm{X}$ & $(-)$ & $(-)$ \\
\hline $\begin{array}{l}\text { Übertragungs- } \\
\text { intervall }^{3}\end{array}$ & $\begin{array}{l}\text { Zielwert (s); } \\
\text { Maximal- und } \\
\text { Minimalwerte (s) }\end{array}$ & $\mathrm{X}$ & $\mathrm{X}$ & - \\
\hline
\end{tabular}

\footnotetext{
${ }^{1}$ Aktualisierungszeit: Zeit zwischen zwei Botschaften, welche vom Kommunikationssystem an die empfangende Anwendung übergeben werden [BZ17]

2 Überlebenszeit: Zeit, welche eine Anwendung nach dem Auftreten eines Kommunikationsfehlers weiter arbeiten kann ohne dass die Kommunikation wieder hergestellt wird [BZ17].

${ }^{3}$ Übertragungsintervall: Zeit zwischen zwei Botschaften, welche von der Anwendung an der Kommunikationsschnittstelle übergeben werden [BZ17].
} 
Weiterhin wurden auch erste Vorschläge für die Schnittstellen von 5G-Zeitsynchronisierungs- und 5G-Lokalisierungsdiensten erarbeitet [3G18b], [3G18f].

\section{G Alliance for Connected Industries and Automation (5G- ACIA)}

Im April 2018 wurde die Arbeitsgemeinschaft „5G Alliance for Connected Industries and Automation (5G-ACIA) " [5G18] im ZVEI in Frankfurt/Main gegründet. Der erste öffentliche Auftritt der 5G-ACIA war auf der Hannover-Messe 2018 [ZV18].

Das Ziel der 5G-ACIA ist es, die bestmögliche Anwendbarkeit von 5G-Technologien und 5G-Netzen für die Industrieautomation, also die Fertigungs- und Prozessindustrie, sicherzustellen. Hierfür werden relevante technische, regulatorische und wirtschaftliche Aspekte adressiert, diskutiert und bearbeitet [5G18].

Abbildung 3 zeigt die Struktur der 5G-ACIA. Die Arbeiten der Arbeitsgruppe 1, Use Cases and Requirements" spiegeln und ergänzen die Arbeiten der 3GPP zu den industriellen Dienstanforderungen, insbesondere zu TR 22.804 (FS_CAV) und TS 22.104 (cyberCAV).

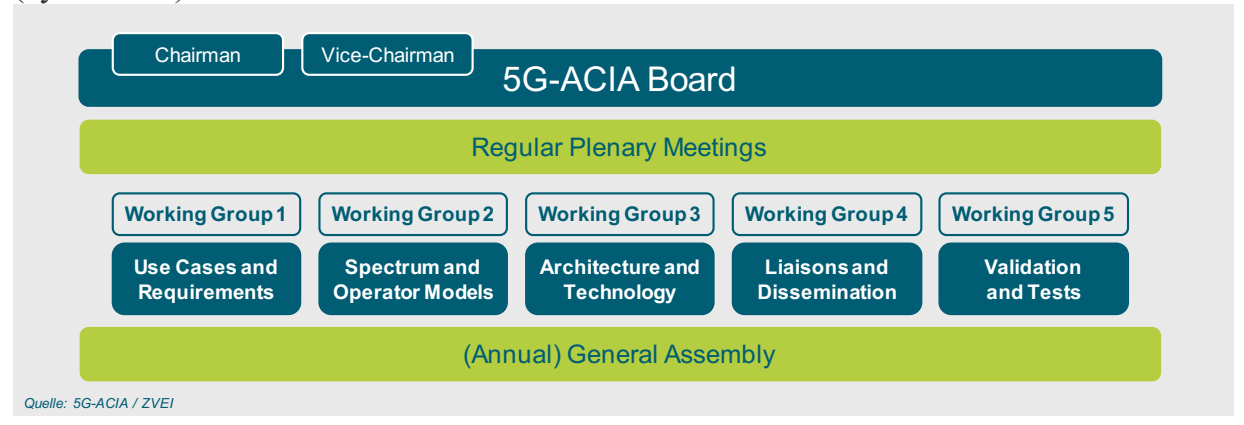

Abb. 3. Struktur der 5G-ACIA

Die 5G-ACIA hat 40 internationale Mitglieder (Stand Oktober 2018) aus dem gesamten, internationalen 5G-Öko-System. Neben den industriellen Nutzern der 5GTechnologie, den Firmen aus der Industrieautomation, der Fertigungs- und der Prozessindustrie, sind auch Netzinfrastrukturanbieter, Mobilfunknetzbetreiber und Chiphersteller aus dem 5G-Umfeld und entsprechende akademische Partner in der 5G-ACIA vertreten. 


\section{Offene Themen}

\subsection{Netzwerkanforderungen}

Um 5G-Systeme zu spezifizieren welche den stringenten Anforderungen der „vertikalen" Kontrollanwendungen entsprechen, müssen die eingesammelten Dienstanforderungen in Netzwerkanforderungen umgesetzt werden. Dies hat sich besonders beim Themenkomplex Ende-zu-Ende-Latenzzeit, Überlebenszeit, Verfügbarkeit und Funktionssicherheit als ein schwieriges Thema für 5G-Ausstatter und die Hersteller entsprechender 5G-Chips herausgestellt. Wie bereits zum Thema Dienste im Abschnitt 4.5 erwähnt, hat die 3GPP bisher nur Netzwerkanforderungen betrachtet und zu den daraus resultierenden Dienstperformanzen keine verbindliche Aussagen gemacht. Im Fall der „vertikalen“ Kontrollanwendungen ist die 3GPP zum ersten Mal mit einem echten Dienstszenario konfrontiert: die Automatisierungsanwendung fordert verbindliche Dienstperformanzen und die 3GPP, ihre Mitglieder und die an Entwicklung, Aufbau und Betrieb von 5G-Netzen Beteiligten müssen während der Spezifikation, der Entwicklung, der technischen Planung und der Aufstellung von industriellen 5G-Netzen sicherstellen, dass diese Dienstanforderungen verbindlich eingehalten werden können. Aufgrund des geschichtlichen Hintergrundes bei der 3GPP und den bisherigen Einsatzgebieten von Mobilfunknetzen muss hier noch weiteres, umfangreiches Wissen erarbeitet werden, wie solche Dienstanforderungen in Netzwerkanforderungen umgesetzt werden können.

$\mathrm{Zu}$ diesem Thema wurden Diskussionen zwischen den „vertikalen“ Interessenten und den Ausstattern gestartet. Erste Ergebnisse sollen im November 2018 veröffentlicht und in TS 22.104 eingebracht werden.

\subsection{Verhaltensweise der Dienste}

Neben der semantischen Beschreibung der Dienstschnittstelle, also der Angabe von an der Schnittstelle auszutauschenden Parametern und deren Syntax, beinhaltet die Beschreibung eines Dienstes auch die Beschreibung des Verhaltens der Schnittstelle („,behaviour model") [OA08]. Zu diesem Thema liegen schon erste Anforderungen vor, zum Beispiel das eine Anwendung eine Bestätigung auf eine Dienstanfrage innerhalb von maximal $100 \mathrm{~ms}$ erwartet, aber insgesamt steckt dieses Thema noch in den Anfängen. Aufgrund des Zeitdrucks in der Freigabe 16 (normative Anforderungen müssen vor 2019 verabschiedet sein), wird dieses Thema voraussichtlich auf Freigabe 17 verschoben werden.

\section{3 „Native Einbindung“6}

Zum Thema „native Einbindung“ von 5G-Systemen in die industrielle Kommunikation wurden bisher Anforderungen zur Unterstützung von Time-Sensitive Networking (TSN) und Ethernet-Anwendungen eingebracht. TSN ist aber eine Zukunftstechnologie, während heute schon viele andere Kommunikationssysteme in Fabriken installiert 
sind (EtherCAT, PROFINET, ...). Es ist noch offen, wie 5G in schon installierte industrielle Kommunikationssysteme integriert werden kann. Dieses Thema wurde schon in der Interessenorganisation 5G-ACIA [5G08] andiskutiert. Auch die Architekturarbeitsgruppe SA2 der 3GPP bearbeitet dieses Thema in einer Studie [5G18h]. Sobald die Anforderungsspezifikationen in Freigabe 16 abgeschlossen sind, wird dieses Thema bei 5G-ACIA in den Vordergrund rücken.

\subsection{Frequenzzuweisung}

Die Zusicherung von stringenten Kommunikationsdienstanforderungen ist natürlich nur möglich wenn für lokale industrielle 5G-Netze eigene Frequenzbänder zugewiesen werden.

Die Bundesnetzagentur (BNetzA) hat zum Thema Vor-Ort-Versorgung mit hoher Dienstgüte bei der laufenden Vergabe von 5G-Frequenzbändern im Bereich von 3,73,8 GHz erstmals auch die Vergabe von lokalen und regionalen Frequenzen vorgesehen [BN18a]. Der derzeitige Vorschlag sieht vor, dieses 5G-Frequenzband in Bereiche für regionale und lokale Nutzung aufzuteilen, sowie zwischen Nutzung im Innen- und Außenbereich zu unterscheiden. Diese Frequenzbänder sollen in einem offenen Verfahren vergeben werden, so dass zum Beispiel Fabriken mit ihren Produktionsanlagen hiermit eine Möglichkeit erhalten, mit vertretbaren Investitionen lokale Lizenzen zu erhalten, die für anspruchsvolle industrielle 5G-Anwendungen nutzbar sind. Bis zum 28. September 2018 lief eine Anhörung und Kommentierung dieses Frequenzzuteilungsverfahrens und der Nutzungsbedingungen.

Auch im 26-GHz-Bereich sollen Frequenzen für 5G-Anwendungen zur Verfügung gestellt werden. Bis zum 19. Oktober 2018 lief eine Anhörung zu den entsprechenden Erwägungen der Bundesnetzagentur [BN18b].

Wegen der unterschiedlichen physikalischen Eigenschaften (Funkausbreitung, Reichweite, Datenrate, Lokalisierungsgenauigkeit) ist eine Kombination von lokalen Zuteilungen im 3,7-3,8-GHz-Band und im 26-GHz-Band für 5G-Anwendungen nötig, damit sämtliche, wichtige Anwendungsfälle von Industrie 4.0 umgesetzt werden können.

Industrieautomatisierung findet international statt. 5G-ACIA engagiert sich aus diesem Grund bei Frequenzzuteilungen auch auf internationaler Ebene, zum Beispiel bei der ITU-R. 


\section{$7 \quad$ Wie geht es weiter?}

Auf dem Treffen der 3GPP-Arbeitsgruppe SA1 im November 2018 in Spokane, WA (USA) ist geplant, die Dienstanforderungen für 5G in der Freigabe 16 zu finalisieren. Auch die cyberCAV-Spezifikation TS 22.104 ,Service requirements for cyber-physical control applications in vertical domains" soll dann abgeschlossen sein.

Die erarbeiteten Dienstanforderungen für industrielle und vertikale 5G-Netze werden in den weiteren Arbeitsgruppen der 3GPP für die Entwicklung entsprechender 5GArchitekturkonzepte (Arbeitsgruppe SA2) und der entsprechenden technischen Spezifikationen (RAN-Arbeitsgruppen) verwendet, um industrielle 5G-Netze umsetzen zu können, die den hohen Anforderungen von URLLC (sehr geringe Latenz und sehr hohe Verfügbarkeit) und den weiteren Anforderungen an industrielle 5G-Netze genügen.

\section{Literatur}

[3G18] 3GPP, www.3gpp.org

[3G18a] 3GPP SA: Service requirements for the 5G system, 3GPP TS 22.261 V16.5.0, Sophia Antipolis, 2018.

[3G18b] 3GPP SA: Study on Communication for Automation in Vertical Domains, 3GPP TR 22.804 V16.1.0, Sophia Antipolis, September 2018.

[3G18c] 3GPP SA: Feasibility Study on LAN Support in 5G, 3GPP TR 22.821 V16.0.0, Sophia Antipolis, 2018.

[3G18d] 3GPP SA: Study on Business Role Models for Network Slicing, 3GPP TR 22.830 V0.2.0, Sophia Antipolis, 2018.

[3G18e] 3GPP SA1: New WID on Service requirements for cyber-physical control applications in vertical domains (cyberCAV), 3GPP S1-180585, 3GPP TSG-SA Meeting \#80, La Jolla (CA, USA), June 2018

[3G18f] 3GPP SA: Service requirements for cyber-physical control applications in vertical domains, 3GPP TS 22.104 V0.2.0, Sophia Antipolis, August 2018

[3G18g] 3GPP SA1: New WID on Business Role Models for Network Slicing (BRMNS), 3GPP S1-180773, 3GPP TSG-SA Meeting, Gold Coast (Australia), September 2018

[5G18] 5G-ACIA, https://www.5g-acia.org.

[BN18a] Bundesnetzagentur (BNetzA): Anhörung zur lokalen und regionalen Bereitstellung des Frequenzbereichs $3.700 \mathrm{MHz}$ bis $3.800 \mathrm{MHz}$ für den drahtlosen

Netzzugang, 2018,

https://www.bundesnetzagentur.de/DE/Sachgebiete/Telekommunikation/Unternehmen_Institutionen/Freq uenzen/OeffentlicheNetze/RegionaleNetze/regionalenetze.html

[BN18b] Bundesnetzagentur (BNetzA): Anhörung zu ersten Erwägungen für die zukünftige Nutzung des 26-GHz-Bandes (24,25 - 27,5 GHz), 2018, https://www.bundesnetzagen-

tur.de/DE/Sachgebiete/Telekommunikation/Unternehmen_Institutionen/Freq uenzen/OeffentlicheNetze/RegionaleNetze/regionalenetze.html 
[BZ17] BZKI: Aspekte der Zuverlässigkeitsbewertung in ZDKI, Anwendungen, Anforderungen und Validierung im BMBF-Förderprogramm „IKT 2020 - Zuverlässige drahtlose Kommunikation in der Industrie“ (BZKI), 2017

[IE09] IEC: Communication network dependability engineering, IEC 61907, Geneva, 2009.

[OA08] OASIS: Reference Architecture for Service Oriented Architecture Version 1.0, 2008.

[ZV18] ZVEI: Initiative 5G-ACIA nimmt Arbeit auf: Gemeinsam 5G industriefähig gestalten, Pressemeldung, 24. April 2018, Frankfurt/Main, https://www.zvei.org/presse-medien/pressebereich/initiative-5g-acia-nimmtarbeit-auf-gemeinsam-5g-industriefaehig-gestalten/

Open Access Dieses Kapitel wird unter der Creative Commons Namensnennung 4.0 International Lizenz (http://creativecommons.org/licenses/by/4.0/deed.de) veröffentlicht, welche die Nutzung, Vervielfältigung, Bearbeitung, Verbreitung und Wiedergabe in jeglichem Medium und Format erlaubt, sofern Sie den/die ursprünglichen Autor(en) und die Quelle ordnungsgemäß nennen, einen Link zur Creative Commons Lizenz beifügen und angeben, ob Änderungen vorgenommen wurden.

Die in diesem Kapitel enthaltenen Bilder und sonstiges Drittmaterial unterliegen ebenfalls der genannten Creative Commons Lizenz, sofern sich aus der Abbildungslegende nichts anderes ergibt. Sofern das betreffende Material nicht unter der genannten Creative Commons Lizenz steht und die betreffende Handlung nicht nach gesetzlichen Vorschriften erlaubt ist, ist für die oben aufgeführten Weiterverwendungen des Materials die Einwilligung des jeweiligen Rechteinhabers einzuholen. 